\title{
THE EFFECTS OF DIVERSITY ON BUSINESS PERFORMANCE: REPORT OF THE DIVERSITY RESEARCH NETWORK
}

\author{
Thomas Kochan, Katerina Bezrukova, Robin Ely, Susan Jackson, \\ Aparna Joshi, Karen Jehn, Jonathan Leonard, David Levine, and \\ David Thomas
}

\begin{abstract}
This article summarizes the results and conclusions reached in studies of the relationships between race and gender diversity and business performance carried out in four large firms by a research consortium known as the Diversity Research Network. These researchers were asked by the BOLD Initiative to conduct this research to test arguments regarding the "business case"for diversity. Few positive or negative direct effects of diversity on performance were observed. Instead a number of different aspects of the organizational context and some group processes moderated diversity-performance relationships. This suggests a more nuanced view of the "business case" for diversity may be appropriate. () 2003 Wiley Periodicals, Inc.
\end{abstract}

Introduction

Since 1996, a group of industry chief executives and human resource professionals have been working together under the auspices of a nonprofit organization called the Business Opportunities for Leadership Diversity (BOLD) Initiative to help American corporations learn how to leverage their cultural diversity for competitive advantage. These leaders espouse the now popular "business case" for diversity-the view that a more diverse workforce will increase organizational effectiveness. For them, providing more opportunities for women and minorities is a business imperative. Realizing, however, that they lacked clear evidence to support this view, either within their own organizations or more generally across American industry, these business leaders called for definitive research to assess the diversity-performance link. An initial study commissioned by BOLD found that no organizations were collecting the data needed to assess the effects of their diversity practices on firm performance (Corporate Leadership Council, 1997). Therefore, in 1997, the BOLD Initiative asked a group of researchers from a cross section of universities to design a large-scale field research project to examine the relationships between gender and racial diversity and business performance.

This paper presents our conclusions from this five-year research effort. We believe

Correspondence to: Thomas Kochan, MIT Sloan School of Management, Building \#552, Room 583, Cambridge, MA 02142; telephone: 617-253-6689; e-mail: Tkochan@mit.edu

Human Resource Management, Spring 2003, Vol. 42, No. 1, Pp. 3-21

(c) 2003 Wiley Periodicals, Inc. Published online in Wiley InterScience (www.interscience.wiley.com $)$.

DOI: 10.1002/hrm.10061 
During the 1990s, diversity rhetoric shifted to emphasize the business case for supporting workforce diversity. this to be the largest field-based research project on this topic undertaken to date. We summarize our results here and their implications for managers and human resource practitioners and describe the challenges we encountered along the way in the hopes of advancing the study and practice of diversity in organizations in the future.' Our results suggest the need to move beyond the business-case argument for advancing the practice of diversity in industry and how to modify this argument to reflect the complexities we discovered in our research. We also propose steps that industry professionals can take to leverage the potential benefits of diversity and to strengthen their ability to assess diversity initiatives in their organizations.

\section{Historical Context of the Business-Case Perspective}

The recognition that diversity is a reality in the workforce has generated an enormous amount of activity over the years among leaders in business, government, and civil society alike (for an extended discussion, see Jackson \& Joshi, 2001). An outcome of the civil rights movement, Title VII of the 1964 Civil Rights Act, made it illegal for organizations to engage in employment practices that discriminated against employees on the basis of race, color, religion, sex, and national origin (age and disability were legislated after 1964). Through these government actions, society made a statement: employers must provide equal employment opportunities to all people of similar qualifications and accomplishments. In addition, Executive Order 11246, issued in 1965, required government contractors to take affirmative actions to overcome past patterns of exclusion or discrimination. These societal mandates curtailed formal policies that discriminated against certain classes of workers and raised the costs to organizations that failed to implement fair employment practices. The laws remain a part of the legal responsibilities under which firms and other labor-market institutions, such as unions or job-matching organizations, operate today.

By the late 1970s and into the 1980s, there was growing recognition within the pri- vate sector that, while the legal mandates were necessary, they were not sufficient for ensuring the effective management of diversity within organizations. Although the workforces of many organizations became more diverse, entrenched organizational cultures, which remained inhospitable to traditionally underrepresented groups, were slow to change. To promote the development of more positive organizational cultures that would support the effective development of a more diverse workforce, many companies and consulting firms began to offer training programs aimed at "valuing diversity". These efforts focused on changing employees' attitudes and eliminating behaviors that reflected more subtle forms of discrimination and exclusion, which often inhibited effective interactions among people. The widespread adoption of such training programs expanded the concept of "diversity" as people began to realize that visible, legally recognized, demographic differences such as race and gender were not the only types of differences that affected work relationships among employees. Gradually, training initiatives proliferated, encouraging employees to value the wide range of physical, cultural, and interpersonal differences, which would presumably enhance decision making, problem solving, and creativity at work. Unfortunately, however, most studies show that such training rarely leads to the desired long-term changes in attitudes and behavior (Bezrukova \& Jehn, 2001).

During the 1990s, diversity rhetoric shifted to emphasize the business case for supporting workforce diversity. Figure 1 reports how the former CEO of Hewlett Packard described the new rhetoric. Essentially, he was looking for a way to convince his fellow executives and managers that to manage diversity effectively is a business necessity not only because of the nature of labor and product markets today, but also because a more diverse work force-relative to a homogeneous one-produces better business results. He believed that providing evidence to support these claims would accelerate the rate of progress employers would make in hiring and developing a more diverse workforce and produce organizations that are more fully integrated across occupations and levels of hierarchy. Likewise, for diversity advocates, 
The Business Case for Diversity

"I see three main points to make the business case for diversity:

I. A talent shortage that requires us to seek out and use the full capabilities of all our employees.

2. The need to be like our customers, including the need to understand and communicate with them in terms that reflects their concerns.

3. Diverse teams produce better results.

This last point is not as easy to sell as the first two-especially to engineers who want the data. What I need is the data, evidence that diverse groups do better."

Figure 1. Lew Platt, former CEO of Hewlett Packard, comments to the Diversity Research Network, Stanford Business School, March 18, 1998.

the new imperative was to find evidence to support the business-case argument.

In fact, as both the study commissioned by BOLD and our own reviews of the research literature (see, e.g., Richard \& Johnson, 1999; Richard et al., 2002; Williams \& O'Reilly, 1998) have shown, there is little research conducted in actual organizations that addresses the impact of diversity or diversity-management practices on financial success. While there are a large number of laboratory experiments that test specific diversity-performance hypotheses, there are few such studies in real organizations and fewer still that assess this hypothesis using objective performance measures. An exception is a study that compared companies with exemplary diversity-management practices to those that had paid legal damages to settle discrimination lawsuits. The results of this study showed that the exemplary firms also performed better as measured by their stock prices (Wright et al., 1995). Overall, however, the search for evidence that directly supports the business-case hypothesis has proved elusive.

Two reasons might explain this lack of evidence. First, diversity is extremely difficult to study in organizational settings be- cause it raises sensitive issues that are difficult to discuss. In addition, organizations, including many we contacted during this project, are reluctant to share their experiences or data, given the legal climate and the potential for litigation. Another reason for the lack of evidence linking workforce diversity to business performance may be that the relationship between diversity and the bottom line is more complex than is implied by the popular rhetoric. Decades of research on the effects of diversity within teams and small groups indicate that diversity can have negative effects, as well as positive ones. The empirical literature does not support the simple notion that more diverse groups, teams, or business units necessarily perform better, feel more committed to their organizations, or experience higher levels of satisfaction (Jackson et al., 1995; Millikin \& Martins, 1996; Williams \& O'Reilly, 1998). Instead, the evidence suggests that diversity may simultaneously produce more conflict and employee turnover as well as more creativity and innovation (Jehn et al., 1999; cf. Williams \& O'Reilly, 1998). For example, this pattern of mixed results was found in two studies that examined diversity within top management teams in the banking industry. In one study, diversity in top management teams was associated with greater innovation within bank branches (Bantel \& Jackson, 1989). In another, diversity also associated with higher rates of turnover among top management team members (Jackson et al., 1991). Thus, the research literature paints a more complex picture of the consequences of diversity than does the popular rhetoric.

\section{The Diversity Research Network and Research Project}

It was this mismatch between research results and diversity rhetoric that led us to agree to form a Diversity Research Network. Our purpose was to conduct a multi-firm study of the effects of racial and gender diversity on organizational performance. The Alfred P. Sloan Foundation, the Society of Human Resource Management (SHRM) Foundation, and the BOLD Initiative provided the funding for the Network's research. 
Figure 2 presents the model that guided the design of the studies discussed in this article. Members of the Diversity Research Network developed the model collaboratively based on a comprehensive review of the large number of laboratory studies and the small number of field studies concerning the effects of diversity on group dynamics and group performance (Richard et al., 2002; Williams \& O'Reilly, 1998). The model reflects both theory and empirical research, which suggest that whether diversity has a positive or negative impact on performance may depend on several aspects of an organization's strategy, culture, and human resource (HR) practices. In addition, the model proposes that these effects are likely to operate through group or team processes, such that under facilitating conditions, diversity is associated with positive group or team processes and is therefore beneficial to performance; whereas under inhibiting conditions, diversity is associated with negative group or team processes and is therefore detrimental to performance.

Specifically, the literature suggests that diversity, if unattended, is likely to have an adverse effect on group processes, such as communications, conflict, and cohesion (Williams \& O'Reilly, 1998). More specifically, diversity in a work group can produce lower cohesion and miscommunication among group members, which can lead to group conflict (Jehn, 1995). Some of this conflict may be productive-if, for example, it avoids "groupthink" and brings additional points of view into the discussion-whereas other forms may worsen group performance.

The connections shown in the model that link diversity to team processes and then to performance seem logical, but we recognize that past research has not always found strong linkages between diversity and performance outcomes. In fact, past research suggests that there may be no direct positive or negative relationship between diversity and performance outcomes. In some groups, diversity may improve performance, while in other groups, diversity may be detrimental to performance (Jackson, 1992; Jehn et al., 1999; O'Reilly \& Flatt, 1989; Richard, 2001; Steiner, 1972). If diversity has inconsistent effects across groups, then in studies that examine the relationship between diversity and performance

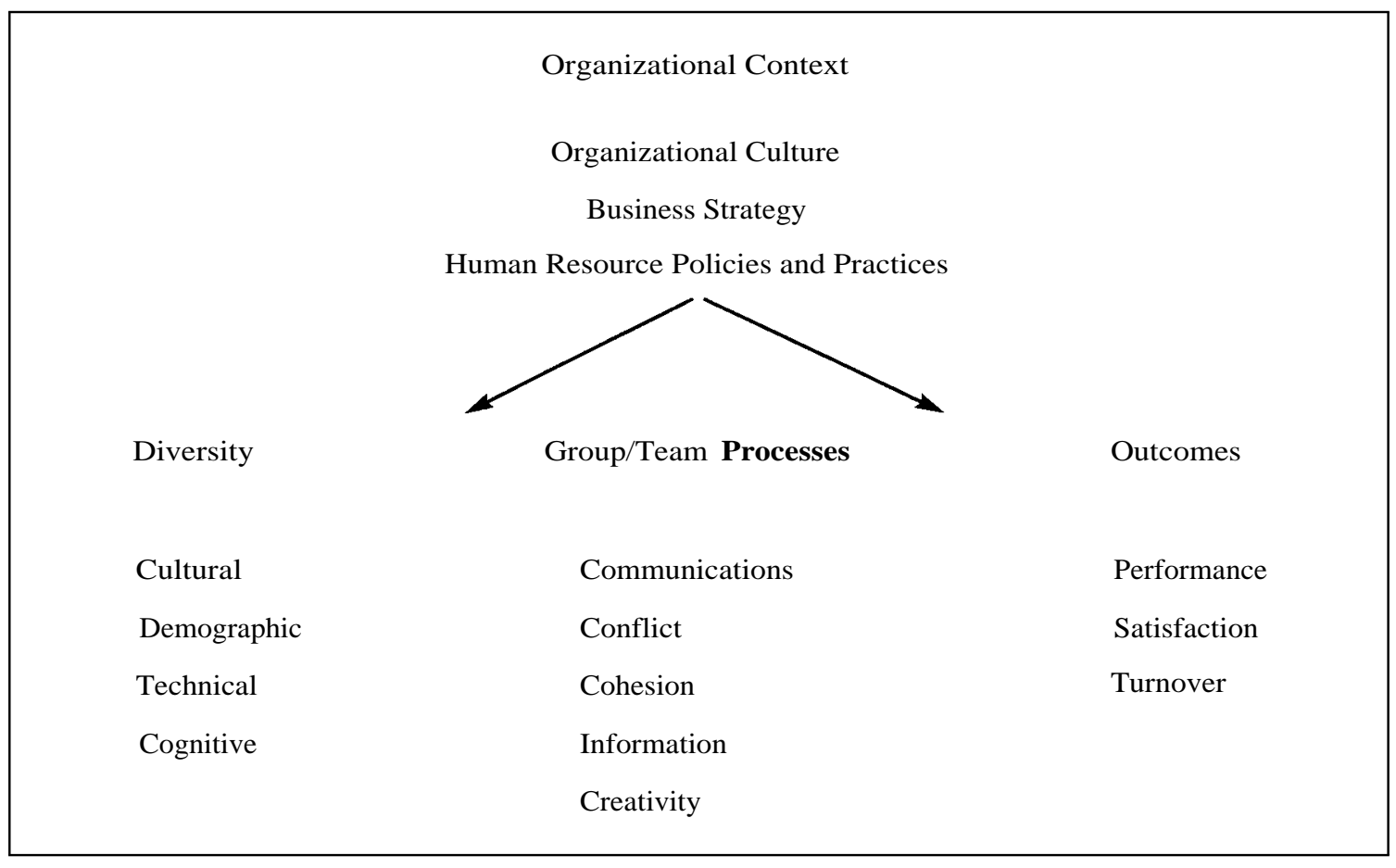

Figure 2. The model: the effects of diversity on group processes and outcomes. 
across many groups, the positive and negative effects may cancel each other out so that no effect obtains. Therefore, our research model suggests that the relationship between diversity and performance may depend on the organizational context in which the work takes place. For example, the effects of diversity on organizational performance might be more favorable if group leaders and members build on team members' creativity and information. Diversity may also be more likely to improve performance when group members and leaders are trained to deal with group process issues, particularly those involved in communicating and problem solving in diverse teams. Presumably, HR practices for recruiting, selecting, training, motivating, and rewarding employees partially determine whether team members and leaders are skilled in communicating with and coordinating among members of diverse teams. When HR practices support the creation of a workforce that has the skills needed to turn diversity into an advantage, diversity is more likely to lead to positive performance outcomes. In other organizations, however, HR practices may inadvertently result in teams that are diverse but unskilled in diversity management. Such organizations are more likely to experience negative outcomes, such as disruptive conflict and increased turnover, which can harm performance.

Although not shown in the model, there is another hypothesis implicit in the business case for diversity, which is related to the second point in Figure 1: a match between the demographic composition of the workforce and the firm's customer base will yield higher sales, thus enhancing organizational performance. The evidence bearing on this hypothesis is limited, however, and derived largely from laboratory studies. One of the four studies included in this project contains what we believe to be the largest and most complete examination of this hypothesis to date.

\section{Data Collection}

The BOLD Initiative first approached several of us in early 1997 with the idea of forming a collaborative industry-university research project to explore the business case for diversity. Researchers from a number of uni- versities met twice to discuss whether such a project was feasible. We were well aware of the difficulties associated with field research on this topic. It raises politically and emotionally charged issues, as well as legal concerns. In addition, it would be difficult to develop and even more difficult to implement a research design that would enable us to draw valid, convincing conclusions about the causal effects of diversity on organizational outcomes. In an ideal world, such a design would entail longitudinal data collected from a large and representative sample of organizations, enabling us to track how changes in demography influence performance over time. We knew from past organizational research that the data needed to examine the impact of diversity on performance are quite extensive, seldom collected, or relatively inaccessible, and unlikely to be comparable across organizations. Yet we all shared the view that if we were to generate knowledge that would be useful to practitioners on the relationship between diversity and performance, we needed to move the research on this topic from the laboratory to the field. Moreover, we were intrigued by the potential benefits of forming a research network among those working on this topic, generating a common framework to guide research, and comparing results across multiple organizations. Some of us had had positive experiences in building and participating in a similar network to explore the relationship between human resource practices and firm performance, a topic that individual researchers had previously found equally difficult to study (Cohen, 1998; Ichniowski et al., 1998). Therefore, we decided to move ahead with the support of the Sloan Foundation, SHRM, and the BOLD Initiative.

The process of recruiting companies to provide the data and participate in the research proved to be even more difficult than we had expected. Over a two-year period (1998-2000), members of the research network and leaders of the BOLD Initiative initiated discussions with over 20 large and wellknown Fortune 500 companies, all of which expressed considerable interest in the topic. However, after often considerable discussion of the data, confidentiality, and time commit-
When HR practices support the creation of a workforce that has the skills needed to turn diversity into an advantage, diversity is more likely to lead to positive performance outcomes. 
While our original hope was to collect the same kind of data in each company and to use the same instruments for measuring the group process and context variables in our model, it quickly became apparent that this was not feasible. ments, all but four companies declined to participate. In some cases, the diversity advocates and professionals in the company lacked sufficient influence to convince line managers to spend the time required to collect the necessary data. In other cases, these professionals were reluctant to examine the effects of their organization's policies, with the view that they had sufficient top management support for their current initiatives and did not need to demonstrate a business case to maintain this support. In still other cases, we found that, even with the support of the CEO, objections raised by internal or external legal counsel and/or by other managers who would need to provide the data proved insurmountable. In the end, each of the firms that agreed to participate had a prior relationship with one or more members of the research team and/or leaders of BOLD Initiative and, therefore, had already established a high level of trust. Thus, the first lesson of this research was that not only had none of the organizations we contacted ever conducted a systematic examination of the effects of their diversity efforts on bottom-line performance measures, very few were interested in doing so.

While our original hope was to collect the same kind of data in each company and to use the same instruments for measuring the group process and context variables in our model, it quickly became apparent that this was not feasible. Each company had its own particular ways of collecting and storing human resource data and three of four firms indicated a strong preference for using their own internal survey measures to capture the variables in the model. Therefore, each study draws on somewhat different kinds of data to address common questions about the effects of diversity on performance. All four companies are large and highly respected, and each has a long history of success in achieving a diverse workforce and commitment to leveraging diversity to enhance organizational performance.

Overall, our conclusions are based on analyses of a mix of qualitative and quantitative data collected across the four studies. Within each company, we identified comparable teams, work groups, or business units to serve as the unit of analysis. In one study, we collected qualitative data on businessunit cultures, HR and managerial practices, and business strategies and on the quality of business-unit processes. In others, we relied on survey data to assess these aspects of the organizational context and the quality of group processes. In all four studies, we used archival data on the demographic composition of teams, work groups, or business units; in one study, we also had census data on the demographic composition of the communities from which the business units drew their customers. And finally, in all four companies, we used a variety of objective measures to assess performance.

\section{Results}

\section{Study 1: A Large Information-Processing Firm}

Study 1 was conducted by the research team of Karen Jehn and Katerina Bezrukova from the Wharton School at the University of Pennsylvania. This study is based on data from a large Fortune 500 informationprocessing company with over 26,000 employees. Diversity has been at the forefront of this company's social and business agenda for over half a century. In the early 1940s, when the company chairman took several sales representatives, including one African American, to an awards ceremony, the hotel hosting the event refused to register the African American, so the chairman left with his sales force in tow. In 1987, management realized that many minority employees were not advancing through the ranks, so the Women's Resource Group and the Minority Resource Group were created to address the issue. In 1992, a Diversity Task Force was created to develop a strategic plan for promoting diversity. Each year since then the Diversity Leadership Council, comprised of employees at every level and in every department of the company, helps to create diversity action plans, which outline initiatives linked to the strategic goals of the Diversity Task Force.

Each business unit of the company is required to submit an end-of-year report measuring how well the unit performed against its diversity objectives. This report details 
quantitative information on diversity initiatives, including the hiring and promotion of women and minorities, succession planning, development, retention, and training. This approach has resulted in a number of tools designed to support the company's diversity objectives. For instance, all business-unit newsletters cover diversity, employees have access to a lecture series on diversity, and "Managing Diversity" is taught at every manager orientation session.

The company's external practices are also shaped by their belief that diversity is essential to innovation and growth. These strategies include establishing alliances with external women's and minority organizations, supporting small businesses, and supporting regional minority business councils across the country. Additionally, the company actively supports minority scholarships, cooperative education, and internship programs; and it provides substantial funding to national organizations concerned with professional, social, and educational goals in minority communities.

To generate measures of the group process and context variables, we content analyzed qualitative data contained in company documents that were part of a human resources-sponsored program created to identify high potential employees and to recognize and hold accountable leaders at all levels of management and supervision. As part of this program, managers create Development Plans (DPs) for their work groups and discuss these plans with their supervisors. The Plans capture the dominant group processes occurring in groups. In addition to DPs, managers and supervisors evaluate their leadership competencies (i.e., values, goals, skills, and knowledge). The competencies assessed in the supervisor reports serve as indirect evidence of the actual context regarding organizational cultures, business strategies, and human resource practices (Dozy et al., 1993).

To specify our group process and context variables, we developed respective lists of key words based on relevant group and organizational theories as well as the concepts used in the company's rhetoric. Teamfocused processes relating to building com- mitment and group spirit, change-focused processes relating to innovation and exploring new perspectives, and career-focused processes relating to career advancements and professional success exemplify constructive group processes we investigated in this study. Context variables are defined by business strategies (i.e., growth, stability, and customer-oriented), culture (i.e., people- and competition-oriented), and human resource practices (i.e., training- and diversity-oriented). Following the method of Jehn and Werner (1993), which was successfully employed in past organizational research, two independent raters reviewed the surrounding context and coded the textual data for each work group on each of these dimensions. Two measures of team performance were available for this study: average performance-appraisal ratings provided by the managers of each business unit and average bonuses of team members. Using these measures, our test of the model guiding the overall research project revealed the following:

1. There were no significant direct effects of race or gender diversity on either team performance-appraisal ratings or bonuses.

2. Diversity had a significant effect on group processes, but the nature of the effect depended on whether the diversity was in gender or race. Specifically, gender diversity increased constructive group processes, while racial diversity inhibited them.

3. Training- and development-focused HR practices, including coaching, open communications and interactive listening, and providing challenging assignments and opportunities for development, reduced the negative effects of racial diversity on constructive group processes.

4. Diversity-focused HR practices enhanced the positive effects of gender diversity on constructive group processes.

5. Constructive group processes, in turn, had a positive impact on performance ratings and group bonuses.
To specify our group process and context variables, we developed respective lists of key words based on relevant group and organizational theories as well as the concepts used in the company's rhetoric. 
To ensure that their workforce reflects the communities they serve, the company aggressively recruits candidates of all backgrounds.
We also conducted additional analyses on a larger sample of groups for which no group process data were available. With this sample, we examined the role that organizational context played in shaping the relationship between diversity and performance. We found the following:

6. Gender diversity was positively associated with group bonuses in business units with a people-oriented organizational culture, diversity-focused human resource practices, and customer-oriented business strategy but not in units that lacked those specific cultures, practices, and strategies.

7. Racial diversity was negatively related to performance in business units with competitive organizational cultures, growth-oriented business strategies, and training-focused human resource practices but not in units that lacked those specific cultures, practices, and strategies.

In summary, our results in this organization showed no significant direct effects of either racial or gender diversity on performance. Gender diversity had positive effects on group processes while racial diversity had negative effects. The negative relationship between racial diversity and group processes was, however, largely absent in groups that had received high levels of training in career development and diversity management.

\section{Study 2: A Financial Services Firm}

Robin Ely and David Thomas at the Harvard Business School carried out Study 2. The data come from the retail branches of a large financial services firm that is highly respected for integrating a commitment to diversity into its managerial policies and strategies. At the corporate level, the company has implemented four practices in particular which they believe represent "best practice" in the field. First, all senior managers in the company, including regional managers of the retail branches, are held accountable for managing a formal diversity plan and for linking diversity to education, recruiting, succession planning, career development, and business growth. These plans cascade down to individual branch managers who, as part of their regional manager's plan, have diversity objectives they are required to meet. Second, in addition to a company-wide Diversity Council, chaired by the CEO, each business hosts its own diversity council chaired by its respective business executive, ensuring hands-on employee involvement in their diversity initiatives. The company now has 45 diversity councils around the world, involving some 1000 employees, including many retailbranch employees. Third, the company considers itself unique in extending the focus of its diversity efforts beyond race and gender. Their education efforts and dialogue with employees across the company include race, gender, disability, religion, sexual orientation, and age. Finally, they have an aggressive construction plan for back-up childcare centers with the belief that they need to support the diverse work-life needs of their employees. They are currently constructing a network of 17 on-site back-up childcare centers in major company sites across the United States, to which most retail-branch employees have access.

To ensure that their workforce reflects the communities they serve, the company aggressively recruits candidates of all backgrounds. In their entry management training programs, they have established close recruiting ties with historically Black colleges. They participate in a number of internship programs that provide opportunities for both high school and college level students. Their intercept programs include A Better Chance, Smart Start, a Fellows Program, and Summer Jobs for Youth, with the hope that many of their interns will choose full-time employment with their firm upon graduation. Due at least in part to the success of these efforts, there are two distinctive features of the branches' demographic composition. First, the racial composition of the branches is wide ranging, including branches that are predominantly Black, Hispanic, Asian, or White as well as branches with virtually every possible mix of these groups. Thus, this study overcomes a common limitation to existing research on diversity in which racial heterogeneity is often confounded with the 
proportion of minorities, and comparisons are limited to Whites and Blacks, or Whites and "others" (Williams \& O'Reilly, 1998). Second, the branches in this bank are largely female dominated. The male-dominated setting, typical of many corporations, is nonexistent in the retail sector of the bank. This means that the sex composition of the branches is narrow, ranging from all women to about equal proportions of women and men, and that a more diverse branch is one that has a greater than average proportion of men employees, or, stated another way, a sex composition that is closer to a 50:50 ratio of men to women, relative to one that is predominantly female. Our sample consisted of 480 retail branches of the firm located primarily in a large city in the northeastern United States. Branches in this sample ranged in size from 4 to 70 employees, with an average of 15 and a standard deviation of 10. The average proportion of Whites in the branches was $49 \%$; the average proportion of women was $83 \%$.

The data came from three sources: (1) archival data on the demographic characteristics of each employee in each branch, (2) employee attitude-satisfaction data from an annual employee attitude-satisfaction survey, and (3) branch performance data used to allocate bonuses to branches on a semiannual basis. Most of the results presented here are based on analyses of data collected at the end of 1999; some analyses included data from 2000 and 2001 , as well.

We obtained performance measures from the firm's branch bonus-award system whereby branches are assessed semiannually on six areas of performance, relative to goals set for the branch in each area. The six performance areas were: (1) revenue from New Sales, (2) revenue from growing the Consumer Portfolio (growth over the six-month assessment period in revenue from retail customers), (3) revenue from growing the Business Portfolio (growth over the six-month assessment period in revenue from business customers), (4) Customer Satisfaction (a composite score assessed from independently conducted surveys of approximately 50 randomly selected customers for each branch), (5) number of Qualified Referrals to bank services (referrals by employees from one product to another that resulted in sale to the customer), and (6) Sales Productivity (total revenue from new sales relative to total-salary expense). There was also a Total Performance score, which is generated from a weighted point system that the bank uses to assign bonuses to branches. Averaging across five items from the company's employee attitude-satisfaction survey, we developed a branch-level measure of the quality of a branch's team processes.

As proposed in the model guiding this project, we expected that the relationships between diversity and team processes and between team processes and outcomes would likely depend on contextual factors that differentiated the branches. Using the employee attitude-satisfaction survey, we developed measures of two such factors. First, we examined the proportion of branch employees who had attended at least one of the firm's diversity-education programs. Most of these programs address multiple dimensions of diversity, such as race, ethnicity, gender, age, religion, disability, and sexual orientation. The primary purpose of these programs is to increase awareness o£ cultural differences and how people's perceptions, biases, and stereotypes of others influence their behavior, and teach skills for addressing conflicts and managing discussions of issues related to diversity. We hypothesized that high levels of both gender and racial diversity would lead to more positive outcomes in branches in which a higher proportion of employees had attended at least one diversity program. Second, we developed a measure of the branches' diversity perspectives, a feature of work groups that we identified in previous research as a crucial factor in determining whether racial diversity has a positive or negative impact on group performance (Ely \& Thomas, 2001; Thomas \& Ely, 1996). In particular, we used four items from the survey to develop a measure of the degree to which branches evidenced an integration-andlearning perspective on diversity. In our prior research, we identified three different perspectives on diversity that culturally diverse work groups, or groups aspiring to be culturally diverse, hold. A work group's diversity
The primary purpose of these programs is to increase awareness of cultural differences and how people's perceptions, biases, and stereotypes of others influence their behavior... 
Participation in diversity-education programs did not foster a positive relationship between racial and gender diversity and performance. perspective is the rationale that guides members' efforts to create and respond to cultural diversity in the group. The three perspectives are the discrimination-and-fairness perspective, the access-and-legitimacy perspective, and the integration-and-learning perspective. We found that, whereas all three perspectives can be successful in motivating managers to diversify their staffs, only the integration-and-learning perspective provides the rationale and guidance needed to achieve sustained benefits from diversity. According to the integration-and-learning perspective, the insights, skills, and experiences employees have developed as members of various cultural identity groups are potentially valuable resources that the work group can use to rethink its primary tasks, and redefine its markets, products, strategies, and business practices in ways that will advance its mission. In branches with an integration-andlearning perspective, we expected that employees of all races would be encouraged to bring all relevant insights and perspectives to bear on their work and, hence, that high levels of racial diversity would be associated with better performance in branches that enacted an integration-and-learning perspective on their diversity and worse performance in branches that did not.

The key findings from these analyses are as follows:

1 Across the six separate performance measures and the overall performance index, we found only one significant direct effect of racial diversity on performance: racial diversity was positively associated with growth in branches' business portfolios. Gender diversity had no significant direct effects on any of the performance measures.

2. Our notion that the effects of diversity on performance would depend on certain context variables was partially supported. In particular, racial diversity was associated with higher overall performance in branches that enacted an integration-and-learning perspective on diversity, relative to racially diverse branches that did not enact this perspective and relative to racially homogeneous branches.

3. Employee participation in diversityeducation programs had limited impact on performance. Branches in which a higher proportion of employees had participated in at least one diversity-education program outperformed branches with lower employee participation in these programs on only one measure of performance (sales productivity). Participation in diversity-education programs did not foster a positive relationship between racial and gender diversity and performance. It had no impact on the racial diversity-performance link, and, unexpectedly, a negative impact on the gender diversity-performance link for one measure of performance, such that in branches with high employee participation, greater gender diversity was associated with lower performance.

In summary, this organization has many of the features that should be conducive to leveraging the potential benefits of diversity or, at the very least, mitigating its potential costs. This may explain the direct positive effect that racial diversity had on one measure of performance and the lack of any direct negative effects. Nevertheless, there was room for improvement. Racial diversity had a positive effect on overall performance in branches that used that diversity as a resource for innovation and learning and a negative effect otherwise, suggesting that enacting a learning perspective on diversity can pay off, even in groups embedded within organizations that are already highly committed to and relatively sophisticated about diversity. We expected that employee participation in diversity-education programs might enhance performance, especially in the more diverse branches, but found little evidence to support this. One possible explanation for our finding that, overall, these programs had little impact on performance is that they are serving as an effective ameliorative to problems encountered in more diverse branches, thereby helping to create parity in performance between 
branches with more and less diversity. The inverse relationship between gender diversity and performance in branches with higher employee participation in diversity-education programs is consistent with this interpretation. It may be that branches that are more sex-balanced (i.e., more gender-diverse) are more likely to encounter problems in the first place, relative to their more typical, femaledominated counterparts, thus sending a higher proportion of their employees to these programs. In this case, program participation may more likely signal performance-inhibiting problems than provide performance-enhancing training.

\section{Study 3: An Information-Processing Firm}

Study 3 was conducted by Susan Jackson at the School of Management and Labor Relations of Rutgers University and Aparna Joshi at the Institute of Labor and Industrial Relations, University of Illinois at UrbanaChampaign. The data were drawn from another Fortune 500 firm in the informationprocessing industry. Of all the organizations studied, this company's diversity-management efforts varied least across its different business units or locations.

Over the past several decades, the company consistently promoted workforce diversity and proactively worked to increase the proportions of women and minority employees. It has a long history of supporting employee support/identity groups that provide mentoring and other supports to their members. The company's initiatives for managing diversity are reflected in staffing procedures, performance appraisals, and training. Several national awards have recognized the company's excellent programs for creating and managing diversity.

All divisions of the company must meet annual targets for the representation of majority and minority males and females in each employee grade level. Availability of minority and majority males and females is determined by examining the internal labor pools as well as U.S. Census data. Of the entire work force in the U.S. division, approximately 33\% were women, 17\% were African Americans, less than 10\% were Hispanics, and Asian and other ethnic groups comprised about 5\%. These numbers attest to the success the company has achieved in recruiting and promoting a diverse workforce.

In order to enforce the annual targets described above, performance appraisals for line managers included measures of the managers' abilities to achieve the targets. The performance appraisals were used for making promotion and compensation-related decisions. Training practices included intensive diversity training. Trainers used behavioral modeling techniques to help develop managerial capabilities for interacting with subordinates and colleagues irrespective of demographic differences. Thus the training efforts focused more on skill-building than on building awareness or modifying attitudes.

The quantitative portion of this study focused on the company's U.S. employees in the sales $(n=3970$ employees) and service ( $n=8636$ employees) divisions. The demographic characteristics of employees in sales and service are substantially different. Gender and ethnic diversity was greater in the sales division than in the service division. In service, only $6 \%$ of employees were female, while $35 \%$ of sales personnel were female. Within sales, the ethnic distribution was the same statistically for males and females. Within service, however, the ethnic distribution was statistically different for males and females. Although the difference was small, the pattern shows that females were slightly more likely to be White. The individual participants in this study were organized into 578 sales teams and 1820 service teams. Analyses were conducted separately for these two occupational groups.

The performance measures used in the analyses for the sales teams were sales-team goal achievement and sales-based bonuses. The company recorded performance for individual sales personnel, and from this information we created team-performance measures by averaging across all members of a team. Sales-team goal achievement was the average value of the team sales representatives' performance against their individual goals for generating sales revenue. This measure was a percentage value that reflected the actual revenue generated compared to
The company's initiatives for managing diversity are reflected in staffing procedures, performance appraisals, and training. 
Gender

diversity within regions was positively related to goal achievement as well as speed of response to customers. the targeted revenue. The team sales-based bonus measure was the average dollar amount of monthly bonuses awarded to the team sales representatives. Bonuses were based on individual sales performance relative to revenue goals and were considered by the firm to be a direct indicator of performance for these employees.

For the service teams the performance measures used were the teams achievement of goals related to machine performance and service response times. All service teams were responsible for maintaining reliable machines while utilizing resources effectively. Thus, one measure of team performance used in this company was success in meeting targets for the performance of the machines serviced by the team. Performance targets were set for the team using historical data that took into account the type of machine and the typical performance of such machines. The resulting value was expressed as a percentage. A score greater than $100 \%$ indicated that the quality of repair service was better than the standard and machines assigned to these teams were more reliable. Response time was another measure of team performance used in this company. Response time refers to the amount of time that elapsed from the time a call was received by the team until a technician arrived at a customer site. Response time targets were based on the products that teams serviced and the locations of clients. Response time was expressed as a percentage, with a value over $100 \%$ indicating that the team responded faster than its target.

A summary of results for service teams (see Joshi [2002] for more details) are as follows:

1. Team-level gender and ethnic diversity was not significantly related to process outcomes such as team cooperation.

2. Team gender diversity was not significantly related to team goal achievement. However, there was a significant negative relationship between team ethnic diversity and team goal achievement.

3. In a second round of analyses, we examined the effects of diversity within larger organizational units using hi- erarchical linear modeling techniques. In these analyses, we considered whether diversity had different effects when regions (not smaller work teams) were the focus. Within regions, employees were interdependent with each other, but not everyone was in close personal contact. When we studied regions, our findings changed. Specifically, gender diversity within service regions was positively related to cooperation within the region. Additional analyses revealed that, in service regions with a greater proportion of female service technicians and female managers, teams were more cooperative (regardless of their diversity). We found a similar pattern for performance outcomes. Gender diversity within regions was positively related to goal achievement as well as speed of response to customers. The story does not end here, however.

4. In a third set of analyses, we asked whether the regional demographic context in which teams worked influenced whether the teams benefited from their diversity. We reasoned that diverse teams would be best able to leverage their resources when the members of the team could form beneficial relationships with other employees in the region. Our analyses examined the influence of service region peer and managerial diversity on the relationship between team diversity and team process and performance. Our results indicated that the effects of team diversity were influenced by diversity within the region. For regions with greater gender diversity, there was a stronger positive relationship between team gender diversity and team cooperation. That is, gender diverse teams were more likely to be cooperative in regions that also were gender diverse. Similar analyses improved our understanding of the relationship between team ethnic diversity and team goal achievement. In regions 
with little ethnic diversity, the negative relationship between ethnic diversity and goal achievement was moderately strong. However, in regions with more ethnic diversity, the negative effects of ethnic diversity were reduced. Furthermore, we found that ethnically diverse teams performed better when they were embedded in ethnically diverse organizational units.

A summary of results for sales teams (see Jackson \& Joshi [2002] for more details) show the following:

1. For sales teams, we again examined the direct relationship between each indicator of team diversity (e.g., gender diversity and ethnic diversity) and both team processes and team performance. We found no evidence for a direct relationship between team gender or ethnic diversity and the team processes or performance. However, further analyses revealed that tenure diversity moderated the effects of gender and ethnic diversity. For example, in teams with little tenure diversity, ethnic diversity was negatively related to team performance, but in teams with greater tenure diversity, there was a positive relationship between ethnic diversity and team performance.

2. A second set of analyses considered the role of the demographic characteristics of team managers. We found that team performance was influenced by the combination of manager demographics and team diversity. For example, for teams led by male managers there was no relationship between team gender diversity and team performance. However, for teams led by female managers greater gender diversity was associated with poorer performance. In contract, for teams led by managers of color, we found a positive relationship between team ethnic diversity and team performance.
3. As we did for service teams (see \#4, above), we also examined the effects of regional diversity. For sales teams, we again found that regional diversity influences the effects of team diversity. However, the findings for sales teams did not mirror the findings for service teams. For the sales teams, we found a negative relationship between team ethnic diversity and team performance for teams located in regions with greater ethnic diversity. This finding suggests that in a sales setting the ethnic diversity may have some problematic outcomes unless managed effectively. In analyses that considered the combined effects of gender, tenure, and ethnic diversity, we found a similar pattern. That is, in regions with more total diversity (gender, ethnic, and tenure), greater team diversity was associated with poorer team performance.

In summary, this study found no significant direct effects of racial or gender diversity on team performance, but the results do reinforce the argument that organizational context matters. The demographic makeup of the larger organization within which teams function has important consequences for effects of diversity within the team. Although this general conclusion was supported by our analyses for both sales and service teams, we cannot draw sweeping generalizations about the relationships we studied because different patterns of results were found in these two occupational groups.

\section{Study 4: A Large Retail Company}

David Levine and Jonathan Leonard at the University of California-Berkeley Hass School of Business and Aparna Joshi from at the University of Illinois conducted Study 4. The data are drawn from a large retailer with locations across the country. This study asked whether workforces that reflect the racial makeup of the communities in which they are located perform better than those that do not reflect the makeup of the community. It also examined whether diversity
We found no evidence for a direct relationship between team gender or ethnic diversity and the team processes or performance. 
These diversity proponents fought against employers who claimed their (mostly White) customers cared about the race and gender of the employees who served them. within the workplace had an effect on workplace performance. No data about team processes or interactions among employees were available for this study.

Like most national chains, this firm organizes a subset of these outlets into a single entity that invests heavily in establishing brand image. Most of the nonmanagerial employees at work at any time are visible to the public, literally by looking through a window. These workplaces are located in every U.S. market of any significance. Each workplace typically employs 15 to 40 part-time employees with several full-time managers and assistant managers. The employees work scattered shifts through the week. Thus, employees work with a changing mix of the other employees on the payroll that month. In general, most frontline employees rotate through the several tasks in the store, spending some of their time dealing with customers and other time in support tasks.

This study combined employee-level data on demographics, store-level data on sales, and data from the 1990 U.S. Census on community characteristics. The employee data was the complete personnel records from February 1996 to October 1998. Data were analyzed on frontline workplace employees, dropping workplaces with fewer than ten employees. The performance measure was average sales at a store. A number of employee, store, and community characteristics were included as controls.

The results can be summarized as follows:

1. Contrary to theories of customer discrimination, communities with more Whites, Blacks, Hispanics, or Asians did not buy more from stores with similar employees.

2. The effects of diversity within a store are more complex. Gender diversity had no meaningful effect. At the same time, stores with highly female workforces sold less than more mixed stores.

3. Racial diversity has little effect on sales due to two offsetting effects. On the one hand, the index of racial diversity (the odds that two employees picked at random are the same race) predicts higher sales. On the other hand, stores with more Whites sell more in this chain, and the primary means of increasing diversity is to hire fewer Whites. These two effects roughly cancel each other out.

In summary, this study finds no consistent evidence that most customers care whether the salespeople who serve them are of the same race or gender. These results do not support some recent proponents of diversity who advocate diversity so as to satisfy customers' desires to be served by those who physically resemble them. Such arguments may still hold in other sectors, where relationships last longer and involve a higher level of trust and communication between customers and service providers. The results also did not show any harm or benefits from racial or gender diversity within the workplace. Again, we might see larger beneficial effects in settings when employees have more discretion and autonomy-so that they have more scope to act on their group-specific information. Conversely, the harm might be larger in settings where communication among employees is more important. At the same time, these results are heartening for old-fashioned proponents of workplace integration. These diversity proponents fought against employers who claimed their (mostly White) customers cared about the race and gender of the employees who served them.

\section{Summary and Implications}

The studies reported here were conducted in large firms that have well-deserved reputations for their longstanding commitments to building a diverse workforce and managing diversity effectively. Each of these firms has taken steps to ensure that its formal policies support and reinforce its diversity objectives. While their specific practices vary, our investigation clearly documents the importance and value of firm-wide, diversity-sensitive managerial strategies, human resource policies, and organizational cultures. Despite the variability in industry contexts, specific practices, and the performance measures we examined, our quantitative results are strikingly similar. 
We found that racial and gender diversity do not have the positive effect on performance proposed by those with a more optimistic view of the role diversity can play in organizations-at least not consistently or under all conditions-but neither does it necessarily have the negative effect on group processes warned by those with a more pessimistic view. Most analyses yielded no negative effects on team processes at all, but when racial diversity was shown to have a negative effect, it was mitigated by training and development-focused initiatives. Gender diversity had either no effects or positive effects on team processes. This is consistent with research that has shown that sex balanced groups have more positive interactions than either predominantly male or predominantly female groups (Hoffman \& Maier, 1961; Wood, 1987).

There were few direct effects of diversity on performance-either positive or negative. Our findings suggest that, as we expected, this is likely because context is crucial in determining the nature of diversity's impact on performance. Conditions that exacerbated racial diversity's negative effects on performance included a highly competitive context among teams. Finally, there was some promising evidence to suggest that, under certain conditions, racial diversity may even enhance performance, namely when organizations foster an environment that promotes learning from diversity.

In general, we also found that gender diversity was less problematic than racial diversity. We expect that this may be, at least in part, because in the companies in these studies, women-typically White womentended to be better represented than either men or women of color.

If these studies are representative of other leading companies with similarly strong commitments to diversity, our results may suggest that efforts to create and manage diverse workforces have generally paid off by eliminating many of the potentially negative effects of diversity on group processes and performance documented previously in the literature. Moreover, there appear to be some conditions under which diversity, if managed well, may even enhance performance.
An important goal of this research was to explore the feasibility of conducting research on diversity in organizational settings. Our experience demonstrated how difficult it is to conduct this type of field research and how little analytical attention practitioners pay to these issues in organizations today. Few companies are equipped to assess the impact of their diversity efforts on their performance. One clear implication of our work is that organizations need to do a better job of tracking and evaluating the impact of their strategies for managing a diverse workforce.

\section{Managerial Implications}

What implications do we draw from this work for managers? Given the limited nature of our sample and our findings, it would be inappropriate to propose broad or sweeping implications for managerial action. In the course of this project, however, we discussed the state of practice with managers from more than 20 large, well-known, and highly regarded firms as we sought their involvement in our research. Through these discussions we obtained what we believe is a valid picture of the state of practice in managing diversity in large organizations today. Moreover, while our empirical research is limited to four cases, to our knowledge, this research represents the first effort to test a model relating diversity to performance in multiple firms. Thus, with appropriate caution, we offer the following implications for practice.

Modify the business case. The simplistic business case of the past is simply not supported in our research. Our experience and findings in these companies suggest that those who want to invoke a business case to advance the cause of diversity need to modify the way they frame the argument. They should start by recognizing that there is virtually no evidence to support the simple assertion that diversity is inevitably either good or bad for business. Based on our findings, we propose a more nuanced view, which focuses on the conditions that can leverage benefits from diversity or, at the very least, mitigate its negative effects. Our proposed reframing of the business case for diversity follows.
There were few direct effects of diversity on performanceeither positive or negative. 
Human resource managers and other professionals in charge of diversity efforts should take a more analytical approach in performing their roles.
Diversity is a reality in labor markets and customer markets today. To be successful in working with and gaining value from this diversity requires a sustained, systemic approach and long-term commitment. Success is facilitated by a perspective that considers diversity to be an opportunity for everyone in an organization to learn from each other how better to accomplish their work and an occasion that requires a supportive and cooperative organizational culture as well as group leadership and process skills that can facilitate effective group functioning. Organizations that invest their resources in taking advantage of the opportunities that diversity offers should outperform those that fail to make such investments.

Look beyond the business case. We believe this restatement of the business case accurately reflects both our results and the results from prior laboratory studies. However, our results may offer an even stronger implication. It may be that the businesscase rhetoric has run its course. Diversity professionals, industry leaders, and researchers might do better to recognize that while there is no reason to believe diversity will naturally translate into better or worse results, diversity is both a labor-market imperative and societal expectation and value. Therefore managers might do better to focus on building an organizational culture, human resource practices, and the managerial and group process skills needed to translate diversity into positive organizational, group, and individual results. Our more specific recommendations for doing so follow.

Adopt a more analytical approach. Despite the widespread availability and use of human resource information systems, we found that basic HR data about individuals or groups could not be readily linked to business-level performance data. Unable to link HR practices to business performance, HR practitioners will be limited in what they can learn about how to manage diversity effectively, and their claims for diversity as a strategic imperative warranting financial investments weakened accordingly. Human resource managers and other professionals in charge of diversity efforts should take a more analytical approach in performing their roles. Sophisticated data collection and analyses are needed to understand the consequences of diversity within organizations, and to monitor an organization's progress in managing diversity effectively. Currently, organizations typically assess their diversity efforts by simply comparing attitudes, performance, advancement, pay, and so on, among different groups of employees. These comparisons can be useful, but they are only a first step. Equally important but very different questions are: Under what conditions do work units that are diverse with respect to gender or race outperform or underperform work units that are more homogeneous? What conditions mitigate or exacerbate diversity's potential negative or positive effects?

\section{Support experimentation and evaluation.} More work is needed to design and evaluate specific interventions or experiments aimed at creating a positive link between diversity and performance. Of necessity, we relied on assessing this relationship by examining natural covariations in diversity and performance across groups, but there were many other factors that we could neither measure nor control, which may have influenced our findings and no doubt attenuated the size of true effects. Researchers who are better able to isolate effects by studying them in the controlled setting of the laboratory tend to find larger effects than we observed in the field research on which we reported here. Studies that can better replicate these experimental conditions in real organizational settings would increase control without the artificiality of the laboratory. To conduct such research, however, will require executives to commit to this type of experimentation and learning within their own organizations.

Train for group-process skills. Our results suggest that training programs must help managers to develop the leadership and group process skills needed to facilitate constructive conflict and effective communication. These are challenges that will inevitably arise 
for managers who attempt to make diversity a resource for learning, change, and renewal.

In summary, we believe that progress in both research and organizational practice will come through continuing collaborative efforts between researchers and managers as they design and evaluate new approaches to leveraging workforce diversity. Training programs that improve the skills of managers and team members may be particularly useful, but training alone is not likely to be suf- ficient. Organizations must also implement management and human resource policies and practices that inculcate cultures of mutual learning and cooperation.

This research was conducted in partnership with the BOLD Initiative. Support was provided by the Alfred P. Sloan Foundation and the Society for Human Resource Management Foundation. All views expressed here are solely those of the authors.

THOMAS KOCHAN is the George M. Bunker Professor of Management at the MIT Sloan School of Management. His research focuses on the changing nature of work and employment relationships and their implications for organizational governance and public policies. As Codirector of the MIT Workplace Center he is active in bringing together business, community, labor, and policy making groups to coordinate efforts to better integrate work and family life. His most recent book is Working in America: A Blueprint for the New Labor Market.

KATERINA BEZxuxovA is an affiliated faculty member at the Wharton School of the University of Pennsylvania. Her research focuses on workplace diversity, organizational conflict, and group productivity. Her most recent work investigates the effects of demographic faultlines in diverse workgroups.

ROBIN ELY is an associate professor of organizational behavior at the Harvard Business School. Her research focuses on how organizations can better manage their race and gender relations while at the same time increasing their effectiveness. She is currently exploring learning-the process of improving performance through interactions that advance knowledge and understanding-as a key mechanism through which diversity enhances work group functioning.

SUSAN JACKSON is professor of human resource management and director of the doctoral program in Industrial Relations and Human Resources at the School of Management and Labor Relations, Rutgers University. Her research interests include human resource management systems, work team functioning, and workforce diversity. For more information about this work, visit her web site at www.rci.rutgers.edu/ sjacksox.

APARNA JOSHI is assistant professor at the Institute of Labor and Industrial Relations, University of Illinois at Urbana-Champaign. She conducts research in the area of diversity in teams and organizations and in the area of management of global teams. She currently teaches courses on Managing Workforce Diversity and Training and Development.

KAREN JEHN is professor of management at the Wharton School of the University of Pennsylvania. Her research focuses on the effects of diversity on group processes and on the effects of conflict on group performance.

JONATHAN LEONARD is professor of economics at the Haas School of Business at the University of California, Berkeley. His research focuses on the effects of affirmative action on employment and on the effects of industrial relations practices and activities on performance. 
DAVID LEVINE is a professor at the Haas School of Business at the University of California, Berkeley, where he is also editor of the journal Industrial Relations and Associate Director of the Institute of Industrial Relations. His research focuses on labor markets and workplaces. His recent books are Reinventing the Workplace (1995); The American Workplace: Skills, Pay and Employee Involvement (1999); and Changes in Careers and Wage Structures at Large American Employers (2002).

DAVID THOMAS is the H. Naylor Fitzhugh Professor of Business Administration at the Harvard Business School. His research focuses on the influence of cultural diversity on organizational and individual effectiveness, minority executive development, and strategic human resource management. His most recent book (with John Gabarro), Breaking Through: The Making of Minority Executives in Corporate America, was the 2001 Academy of Management George R. Terry Book Award winner for outstanding contribution to management theory and practice.

\section{REFERENCES}

Bantel, K.A., \& Jackson, S.E. (1989). Top management and innovations in banking: Does the composition of the top team make a difference? Strategic Management Journal, 10 (Special Issue), 107-124.

Bezrukova, K., \& Jehn, K.A. (2001). The effects of diversity training programs. Unpublished manuscript. Philadelphia, PA: The Wharton School, University of Pennsylvania.

Cohen, H. (1998). Studying industries and their people. Perspectives on Work, 2, 13-17.

Corporate Leadership Council. (1997). The state of corporate diversity initiatives. Washington, DC: The Advisory Board.

Doty, D.H., Glick, WH, \& Huber, G.P. (1993). Fit, equifinality, and organizational effectiveness: A test of two configurational theories. Academy of Management Journal, 36, 1196-1250.

Ely, R.J., \& Thomas, D.A. (2001). Cultural diversity at work: The effects of diversity perspectives on work group process and outcomes. Administrative Science Quarterly, 46, 229-73.

Hoffman, L., \& Maier, N. (1961). Quality and acceptance of problem solutions by members of homogeneous and heterogeneous groups. Journal of Abnormal and Social Psychology, 62, 401-407.

Ichniowski, C., Kochan, T.A., \& Olson, C. (1998). Assessing the effects of HR on firms, individuals and society: The role of the HR Network. Perspectives on Work, 2, 18-22.

Jackson, S. (1992). Team composition in organizations. In S. Worchel, W Wood, \& J. Simpson
(Eds.), Group process and productivity (pp. 1-12). London: Sage.

Jackson, S.E., Brett, J.F., Sessa, V.1., Cooper, D.M., Julin, J.A., \& Peyronnin, K. (1991). Some differences make a difference: Individual dissimilarity and group heterogeneity as correlates of recruitment, promotions, and turnover. Journal of Applied Psychology, 76, 675-689.

Jackson, S.E., \& Joshi, A. (2001). Research on domestic and international diversity in organizations. In N. Anderson, D.S. Ones, H.K. Sinangil, \& C. Vishwesvaran (Eds.), Handbook of industrial, work and organizational psychology, Volume 2 (pp. 206-231). Thousand Oaks, CA: Sage.

Jackson, S.E., \& Joshi, A. (2002). Diversity in social context: A multi-attribute, multi-level analysis of team diversity and performance in a sales organization. Working paper, School of Management and Labor Relations, Rutgers University, New Brunswick, NJ.

Jackson, S.E., May, K.A., \& Whitney, K. (1995). Understanding the dynamics of diversity in decision making teams. In R.A. Guzzo and E. Salas (Eds.), Team decision making effectiveness in organizations (pp. 204-261). San Francisco: Jossey-Bass.

Jehn, K.A. (1995). A multimethod examination of the benefits and detriments of intragroup conflict. Administrative Science Quarterly, 40, 256-282.

Jehn, K.A., Neale, M., \& Northcraft, G. (1999). Why differences make a difference: A field study of diversity, conflict, and performance in workgroups. Administrative Science Quarterly, 44, 741-763. 
Jehn, K.A., \& Werner, O. (1993). Hapax Legomenon II: Theory, a thesaurus, and word frequency. Cultural Anthropology Method, 5, 8-10.

Joshi, A. (2002). How does context matter? Examining the process and performance outcomes of work team diversity. Dissertation, Rutgers University, New Brunswick, NJ.

Millikin, F.J., \& Martins, L.L. (1996). Searching \{from?\} common threads: Understanding the multiple effects of diversity in organizational groups. Academy of Management Review 21(2), 402-433.

O'Reilly, C.A., \& Flatt, S. (1989). Executive team demography: Organizational innovation and firm performance. Working paper. Berkeley, CA: School of Business, University of California at Berkeley.

Richard, O.C. (2000). Racial diversity, business strategy, and firm performance: A resourcebased view. Academy of Management Journal, 43, 164-177.

Richard, O.C., \& Johnson, N.B. (1999). Making the connection between formal human resource diversity practices and organizational effectiveness: Beyond management fashion. Performance Improvement Quarterly, 12, 77-96.
Richard, O.C., Kochan, T.A., \& Mcmillan-Capehart, A. (2002). The impact of visible diversity on organizational effectiveness: Disclosing the contents in Pandora's black box. Journal of Business and Management, 8, 1-26.

Steiner I.D. (1972). Group process and productivity. San Diego: Academic Press.

Thomas, D., \& Ely, R. 1996. Making differences matter: A new paradigm for managing diversity. Harvard Business Review, September-October, 79-91.

Williams, K., \& O'Reilly, C.A. (1998). Demography and diversity: A review of 40 years of research. In B. Stave and R. Sutton (Eds.), Research in organizational behavior, Volume 20 (pp. 77-140). Greenwich, CT: JAI Press.

Wood, W (1987). Meta-analytic review of sex differences in group performance. Psychological Bulletin, 102, 53-71.

Wright, P., Ferris, S.P, Hiller, J.S., \& Kroll, M. (1995). Competitiveness through management of diversity: Effects on stock price valuation. Academy of Management Journal, 38, 272-287.

\section{ENDNOTES}

1. The details of our analyses and results are available upon request. 